\title{
Association Between Nutritional Indices and Long-Term Outcomes in Patients Undergoing Isolated Coronary Artery Bypass Grafting
}

Omer Tasbulak ${ }^{1}$, Arda Guler ${ }^{1}$, Mustafa Duran ${ }^{2}$, Anil Sahin ${ }^{1}$, Umit Bulut ${ }^{1}$, Yalcin Avci ${ }^{1}$, Ali R. Demir ${ }^{1}$, Serkan Kahraman ${ }^{1}$, Unal Aydin ${ }^{3}$, Mehmet Ertürk ${ }^{1}$

1. Cardiology, University of Health Sciences, Mehmet Akif Ersoy Thoracic and Cardiovascular Surgery Training and Research Hospital, Istanbul, TUR 2. Cardiology, University of Health Sciences, Konya Training and Research Hospital, Konya, TUR 3. Cardiovascular Surgery, University of Health Sciences, Mehmet Akif Ersoy Thoracic and Cardiovascular Surgery Training and Research Hospital, Istanbul, TUR

Corresponding author: Omer Tasbulak, omertasbulak@hotmail.com

\section{Abstract}

\section{Background}

It is well known that approximately $20 \%$ of patients who undergo cardiac surgery experience weight loss in postoperative period. However, there is a lack of data on postoperative consequences of malnutrition. This study aimed to investigate the relationship between nutritional status and long-term outcomes in patients undergoing isolated coronary artery bypass grafting (CABG).

\section{Material and methods}

A total of 586 patients who underwent isolated CABG in our center between January 2015 and March 2016 were included in this study. The primary outcome was major adverse cardiac and cerebrovascular events (MACCE) defined as a composite of all-cause death, non-fatal myocardial infarction (MI), and stroke. Patients were divided into two groups based on their MACCE outcomes. Prognostic nutritional index (PNI), geriatric nutritional risk index (GNRI), and controlling nutritional status (CONUT) scores were used to show the nutritional status.

\section{Results}

The mean follow-up time of the whole study group was $38.08 \pm 13.4$ months. The follow-up time was $39 \pm 13$ months in patients with mortality, while it was $20 \pm 15$ months in those without mortality. The PNI and GNRI values were lower in patients with major adverse cardiac and cerebrovascular events (MACCE) compared to patients without MACCE. The median CONUT score was higher in patients with MACCE.

\section{Conclusion}

Review began 06/29/2021 Review ended 07/14/2021 Published 07/22/2021

\section{๑) Copyright 2021}

Tasbulak et al. This is an open access article distributed under the terms of the Creative Commons Attribution License CC-BY 4.0., which permits unrestricted use, distribution, and reproduction in any medium, provided the original author and source are credited.

Our study showed that nutritional indices including PNI, CONUT, and GNRI were associated with long-term MACCE and mortality in patients who underwent isolated CABG. The use of these scores in order to predict prognosis in patients treated with CABG seems to be an applicable method in clinical practice.

Categories: Cardiology

Keywords: : coronary artery bypass surgery, prognostic nutritional index (pni), geriatric nutritional risk index (gnri), controlling nutritional status (conut), cabg

\section{Introduction}

Cardiovascular diseases are the leading cause of death and disability worldwide. Coronary artery disease (CAD) takes the lead in this group of diseases [1]. With regard to the treatment of CAD, coronary artery bypass grafting $(\mathrm{CABG})$ emerged as a convenient treatment modality together with medical therapies and percutaneous coronary intervention (PCI). Despite the improvements in percutaneous techniques, CABG still remains the preferred method over PCI especially in diabetic patients with multiple vessels or left main coronary artery disease [2]. It is well known that approximately $20 \%$ of patients who undergo cardiac surgery experience weight loss in postoperative period. The stress response to surgery and other comorbid conditions are underlying factors that provoke weight loss and contribute to the state of malnutrition [3].

Malnutrition is a major public health problem and is estimated to affect approximately $30 \%$ to $70 \%$ of hospitalized patients. It has an adverse effect on the cardiovascular system, immunological system, endocrine system, gastrointestinal system, and on the healing process during the recovery period [4]. There are several nutritional indices that have been developed to determine the nutritional status of an individual. Among them, the controlling nutritional status (CONUT) score [2], geriatric nutritional risk index (GNRI) [3], and prognostic nutritional index (PNI) are the most useful markers which provide simple, effective, and 
objective assessments of nutritional status [4]. According to recent studies, these parameters have been shown to be associated with predictors of mortality and morbidity in patients with prior history of heart failure [5-7]. Studies also confirmed that these parameters were also associated with clinical outcomes in patients with CAD $[7,8]$.

There are some publications investigating the effect of these malnutrition criteria on CAD, and especially on mortality and long-term outcomes after PCI [7-9]. Furthermore, they have been shown to be effective in predicting mortality in patients undergoing CABG. On the other hand, there is a paucity of data in terms of correlation between nutritional indices and long-term outcomes and major adverse cardiovascular events in patients undergoing isolated CABG. This study aimed to demonstrate the predictive value of these malnutrition criteria for adverse cardiovascular events in patients undergoing isolated CABG.

\section{Materials And Methods}

This work was a retrospective observational study that complied with the Declaration of Helsinki and was approved by the appropriate health authorities, independent ethics committees, and independent review boards (Mehmet Akif Ersoy Thoracic and Cardiovascular Surgery Training and Research Hospital Local Ethics Committee; date: 22/12/2020; decision no: 2020/83) in our center.

A total of 586 patients between the ages of 35 years and 80 years, who underwent isolated CABG in our center between January 2015 and March 2016, were included in this study. Patients were analyzed retrospectively. Exclusion criteria included emergency procedures, additional valve surgery, active infection(s), recent major surgical procedure or trauma, previously proved systemic inflammatory disease, malignancy, and presence of end-stage renal and/or liver failures.

Demographic data, clinical variables, and medication use were collected from our institutional database. In addition, comorbidities of the patients such as chronic obstructive pulmonary disease, peripheral artery disease, atrial fibrillation, diabetes mellitus, hypertension, and myocardial infarction were recorded. Blood samples were collected in the early morning after overnight fasting, and blood pressure (BP) was measured upon admission. Patients with blood pressure (BP; $140 / 90 \mathrm{mmHg}$ ) or those receiving antihypertensive drugs were regarded as hypertensive. Dyslipidemia was defined as low-density lipoprotein cholesterol (LDL-C) $>140 \mathrm{mg} / \mathrm{dl}$, high-density lipoprotein cholesterol (HDL-C) $>40 \mathrm{mg} / \mathrm{dl}$, triglycerides $>150 \mathrm{mg} / \mathrm{dl}$, or those receiving drugs for hyperlipidemia.

The primary outcome was major adverse cardiac and cerebrovascular events (MACCE) defined as a composite of all-cause death, non-fatal myocardial infarction (MI), and stroke. Secondary outcomes are also defined as hospitalization owing to heart failure and repeat revascularization. Clinical follow-up included a review of medical charts, telephone contact, and questionnaires sent to patients or their families. Mortality data were collected from the medical records of patients who died or who were treated at our institution, and details and causes of death were obtained from other hospitals to which patients had been admitted. Cardiac death was defined as death from CAD, cardiogenic shock, or sudden death. Non-cardiac death was defined as death from other causes. MI was defined as evidence of myocardial necrosis in a clinical setting consistent with myocardial ischemia. Patients were divided into two groups based on their MACCE outcomes.

\section{Nutritional indices assessment}

Objective nutritional index (geriatric nutritional risk index $\{\mathrm{GNRI}\}$, prognostic nutritional index $\{\mathrm{PNI}\}$, and controlling nutritional status $\{$ CONUT\}) scores were calculated at the time of hospital admission. The GNRI was calculated using a previously reported method: GNRI $=14.89 \mathrm{x}$ serum albumin $(\mathrm{g} / \mathrm{l})+41.7 \mathrm{x}$ body weight (kg)/ideal body weight (kg) [10]. Height and weight were measured within 24 hours before surgery. The ideal body weight was calculated as follows: body height - 100 - (\{body height -150\}/4) for males, and body height - 100 - (\{body height - 150\}/2.5) for females. The PNI was calculated using the formula: $10 \mathrm{x}$ serum albumin $(\mathrm{g} / \mathrm{dL})+0.005 \mathrm{x}$ total lymphocytes (count per $\left.\mathrm{mm}^{3}\right)[11]$. The CONUT score consists of three variables: serum albumin, total cholesterol, and total lymphocyte count. Patients with CONUT scores of 9-12, 5-8, and 2-4 were considered to have a severe, moderate, and mild risk of malnutrition, respectively [12]. Those with a score of $0-1$ were considered to have normal nutritional status. The patients were divided into two groups as those with normal CONUT scores and those with mild - moderate - severe CONUT scores. Body mass index (BMI) was calculated using the formula: $\mathrm{BMI}=$ weight $(\mathrm{kg}) /$ height $(\mathrm{m})^{2}$.

\section{Statistical analysis}

Statistical analysis was performed using the IBM SPSS Statistics for Windows, Version 21.0 software (Armonk, NY: IBM Corp.). The data were expressed as $\mathrm{n}(\%)$ for categorical variables. The Pearson chi-square and Fisher exact tests were performed for categorical variables. After normal distribution was analyzed with the Kolmogorov-Smirnov test, the data were expressed as median (25th and 75 th percentiles) for variables without a normal distribution and mean \pm SD for variables with normal distribution. The Student's t-test was used for comparing quantitative variables with normal distribution, and the Mann-Whitney U test was used for comparing quantitative variables without normal distribution. Univariate and multivariate logistic regression analyses were used to determine the independent predictors of primary endpoints of the study 


\section{Cureus}

(MACCEs). Receiver operating characteristic (ROC) curve analysis was conducted to determine the optimal PNI, GNRI, and CONUT values to indicate mortality and primary endpoints of the study (MACCEs) in terms of both sensitivity and specificity. The survival curve using the PNI, GNRI, and CONUT was analyzed using the Kaplan-Meier method, and statistical assessment was performed using the log-rank test. A p-value < 0.05 was considered statistically significant.

\section{Results}

A total of 586 patients (mean age was 59.5; 78\% of patients were male) with CAD and underwent isolated CABG were included. The mean follow-up time of the whole study group was $38.08 \pm 13.4$ months. The follow-up time was $39 \pm 13$ months in patients without mortality, while it was $20 \pm 15$ months in those with mortality. Additionally, the follow-up time was $38 \pm 14$ months in patients without MACCE, while it was $15 \pm$ 9 months in those with MACCE. Baseline characteristics of the patients categorized by MACCE outcomes are listed in Table 1.

\begin{tabular}{|c|c|c|c|}
\hline & MACCE (-) (n=533) & MACCE $(+)(n=53)$ & p-Value \\
\hline Age & $59.5 \pm 9.2$ & $60.5 \pm 8.0$ & 0.474 \\
\hline Gender (women), n (\%) & $117(22)$ & $13(24.5)$ & 0.667 \\
\hline Chronic obstructive pulmonary disease, $\mathrm{n}(\%)$ & $73(13.7)$ & $8(15.1)$ & 0.778 \\
\hline Peripheral artery disease, $\mathrm{n}(\%)$ & $46(8.6)$ & $10(18.9)$ & 0.016 \\
\hline Atrial fibrillation, $\mathrm{n}(\%)$ & $11(2.1)$ & $1(1.9)$ & 0.703 \\
\hline Smoking, n (\%) & $175(32.8)$ & $26(49.1)$ & 0.018 \\
\hline Diabetes mellitus, $\mathrm{n}(\%)$ & $288(54)$ & $34(64.2)$ & 0.158 \\
\hline Hypertension, n (\%) & $411(77.1)$ & $44(83)$ & 0.325 \\
\hline Previous myocardial infarction, $\mathrm{n}(\%)$ & $90(16.9)$ & $9(17)$ & 0.986 \\
\hline Heart failure with reduced EF, n (\%) & $38(7.1)$ & $7(13.2)$ & 0.099 \\
\hline $\mathrm{EF},(\%)$ & $60(50-60)$ & $55(45-60)$ & 0.060 \\
\hline Stroke, n (\%) & $42(7.9)$ & $1(1.9)$ & 0.081 \\
\hline \multicolumn{4}{|l|}{ Clinical admission, n (\%) } \\
\hline Acute coronary syndrome, $\mathrm{n}(\%)$ & 207 (38.8) & $26(49.1)$ & \multirow{2}{*}{0.147} \\
\hline Stable chronic coronary syndrome, n (\%) & $326(61.2)$ & $27(50.9)$ & \\
\hline Prognostic nutritional risk index & $55.45(52.25-59.4)$ & 43.35 (39.75 - 48.8) & $<0.001$ \\
\hline Geriatric nutritional risk index & $58.64(53.85-64.13)$ & $56.06(50.65-62.09)$ & 0.019 \\
\hline CONUT & $1(0-2)$ & $5(4-6)$ & $<0.001$ \\
\hline \multicolumn{4}{|l|}{ CONUT group, $\mathrm{n}(\%)$} \\
\hline Normal nutrition & 391 (73.4) & $5(9.4)$ & \multirow{4}{*}{$<0.001$} \\
\hline Mild malnutrition & $136(25.5)$ & $15(28.3)$ & \\
\hline Moderate malnutrition & $6(1.1)$ & $32(60.4)$ & \\
\hline Severe malnutrition & $0(0)$ & $1(1.9)$ & \\
\hline
\end{tabular}

\section{TABLE 1: Demographic, clinical, and nutritional characteristics of the patients}

EF: ejection fraction, CONUT: controlling nutritional status, MACCE: major adverse cardiac and cerebrovascular events

Clinical history of the patients was similar in terms of hypertension, diabetes mellitus, previous MI, chronic kidney disease, peripheral arterial disease (PAD), heart failure, chronic lung disease, and stroke. Clinical history was also similar in terms of acute coronary syndrome or stable CAD. Patients with smoking and peripheral arterial disease were higher in the MACCE + group. PNI (MACCE $\{-\}: 55.45$ \{52.25-59.4\} vs. MACCE 


\section{Cureus}

$\{+\}: 43.35\{39.75-48.8\}, p<0.001)$ and GNRI (MACCE $\{-\}: 58.64\{53.85-64.13\}$ vs. MACCE $\{+\}: 56.06\{50.65-$ $62.09\}, \mathrm{p}=0.019)$ values were significantly lower in patients with MACCE compared to patients without MACCE. The median CONUT score was higher in patients with MACCE $(1\{0-2\}$ vs. $5\{4-6\}, \mathrm{p}<0.001)$. The number of patients with normal CONUT score $(391\{73.4 \%\}$ vs. $5\{9.4 \%\}, p<0.001)$ was lower in patients with MACCE. Table 2 provided laboratory findings of the patients.

\begin{tabular}{|c|c|c|c|}
\hline & MACCE - $(n=533)$ & MACCE + $(n=53)$ & p-Value \\
\hline Glucose, mg/dl & $114(96-153)$ & 120 (101-198) & 0.094 \\
\hline Creatinine, mg/dl & $0.9(0.77-1.05)$ & $0.9(0.79-1.1)$ & 0.433 \\
\hline Total cholesterol, mg/dl & $180(143-215)$ & $181(143-224)$ & 0.692 \\
\hline Low-density lipoprotein, mg/dl & $105(76-140)$ & $104(71-143)$ & 0.957 \\
\hline High-density lipoprotein, mg/dl & $40(34-47)$ & $40(35-49)$ & 0.545 \\
\hline Triglyceride, mg/dl & 147 (106-209) & $156.5(103-232)$ & 0.765 \\
\hline Hemoglobin, g/dl & $13.9(12.6-15)$ & $13.6(12.1-14.3)$ & 0.137 \\
\hline Platelet, $10^{3} / \mathrm{mm}^{3}$ & $258(212-313)$ & 251 (205-273) & 0.217 \\
\hline White blood count, $10^{3} / \mu \mathrm{l}$ & $8.49(7.25-10.1)$ & $8.14(7.15-9.68)$ & 0.558 \\
\hline Body mass index, $\mathrm{kg} / \mathrm{m}^{2}$ & $27.85(25.47-31.08)$ & $27.38(24.96-30.12)$ & 0.264 \\
\hline AST, $\mu / l$ & $17(14-23)$ & $17(14-21)$ & 0.369 \\
\hline ALT, $\mu / \mathrm{I}$ & $18(14-25)$ & $18(14-21)$ & 0.358 \\
\hline Albumin, mg/dl & $4.4(4.1-4.64)$ & $3.2(2.8-3.74)$ & $<0.001$ \\
\hline 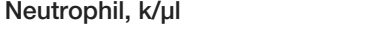 & $5.38(4.34-6.59)$ & $5.03(4.37-6.38)$ & 0.561 \\
\hline Lymphocyte, \% & $2.32(1.88-2.9)$ & $2.33(1.98-2.69)$ & 0.699 \\
\hline
\end{tabular}

\section{TABLE 2: Laboratory findings of the patients}

MACCE: major adverse cardiac and cerebrovascular events, AST: serum aspartate aminotransferase, ALT: alanine aminotransferase

In laboratory parameters, the groups were similar with respect to glucose, creatinine, cholesterol counts, liver enzymes, hemoglobin, and platelet counts. BMI was similar between the groups. Additionally, the nutritional statuses of patients with secondary outcomes were demonstrated in Table 3. 


\section{Cureus}

\begin{tabular}{|c|c|c|c|}
\hline & Secondary outcomes $(-)(n=544)$ & Secondary outcomes $(+)(n=42)$ & p-Value \\
\hline PNI & $55.4(51.83-59.33)$ & $47.1(43.2-51.65)$ & $<0.001$ \\
\hline CONUT & $1(0-2)$ & $3(2-5)$ & $<0.001$ \\
\hline \multicolumn{4}{|l|}{ CONUT group, n (\%) } \\
\hline Normal nutrition & $390(71.7)$ & $6(14.3)$ & \multirow{4}{*}{$<0.001$} \\
\hline Mild malnutrition & $128(23.5)$ & $23(54.8)$ & \\
\hline Moderate malnutrition & $25(4.6)$ & $13(31.0)$ & \\
\hline Severe malnutrition & $1(0.2)$ & $0(0)$ & \\
\hline GNRI & 58.47 (53.81-64.12) & $54.83(52.3-59.92)$ & 0.039 \\
\hline
\end{tabular}

TABLE 3: Nutritional status of the patients when compared to secondary outcomes

PNI: prognostic nutritional index, CONUT: controlling nutritional status, GNRI: geriatric nutritional index

The PNI (55.4 \{51.83-59.33\} vs. $47.1\{43.2-51.65\}, \mathrm{p}<0.001)$ and GNRI (58.47 \{53.81-64.12\} vs. $54.83\{52.3-$ $59.92\}, \mathrm{p}=0.039$ ) values were lower in patients with secondary outcomes compared to patients without secondary outcomes. The median CONUT score was higher in patients with secondary outcomes $(1\{0-2\}$ vs. $3\{2-5\}, \mathrm{p}<0.001)$. The number of patients with normal CONUT score $(390\{71.7 \%\}$ vs. $6\{14.3 \%\}, \mathrm{p}<0.001)$ was lower in patients with secondary outcomes.

ROC curve analyses were conducted to determine the optimal PNI, GNRI, and CONUT cut-off value to indicate mortality and MACCE separately. The highest combined sensitivity and specificity value crossed the curve at 46,97 (sensitivity: 90.5\%; specificity: 91.2\%) for PNI to indicate mortality (Figure 1). 


\section{Cureus}

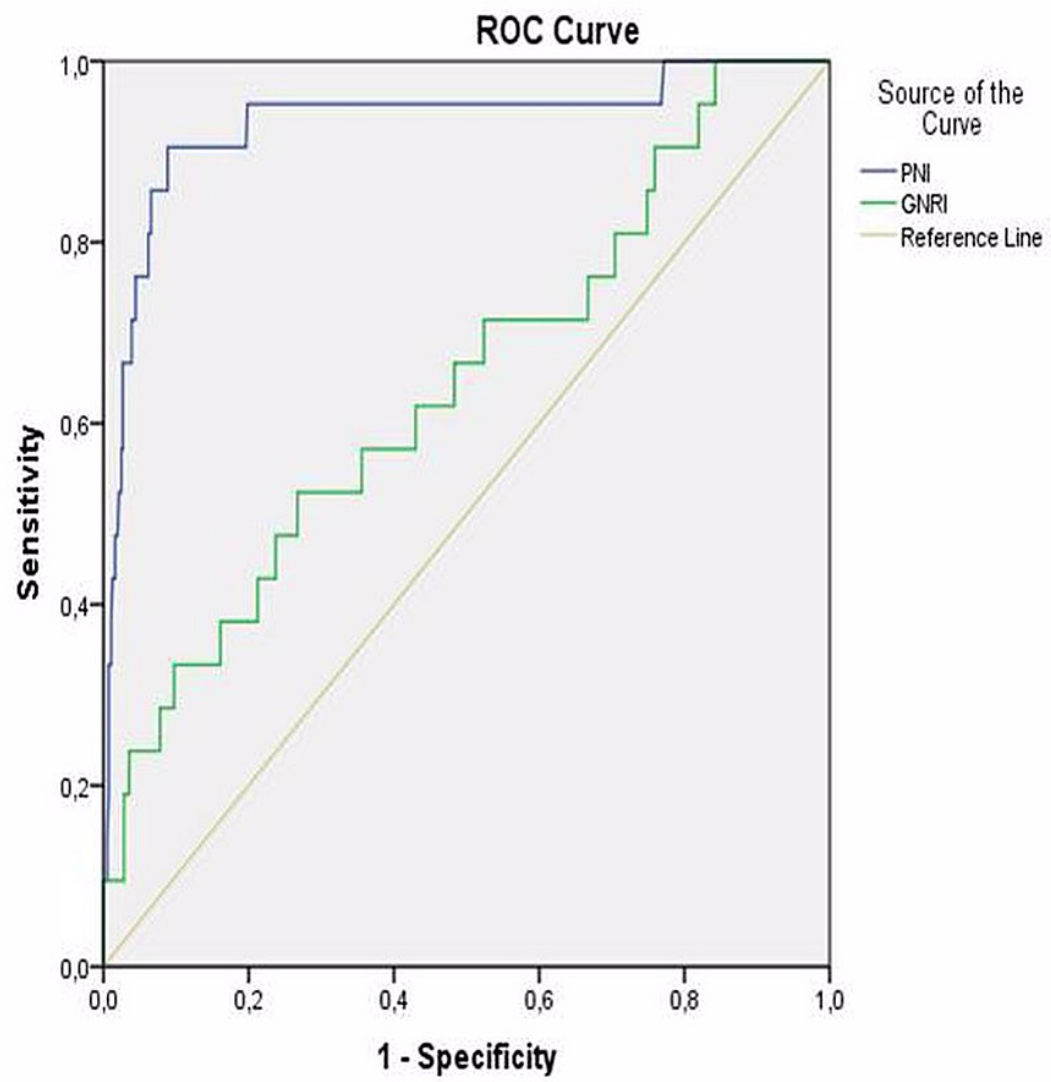

Diagonal segments are produced by ties.

\section{FIGURE 1: ROC curves of PNI and GNRI as a predictor of mortality}

ROC: receiver operating characteristic, PNI: prognostic nutritional index, GNRI: geriatric nutritional risk index

The area under the curve was 0.932 (95\% confidence interval $\{\mathrm{CI}\}: 0.861-1.000 ; \mathrm{p}<0.001)$. The highest combined sensitivity and specificity values crossed the curve at 54.06 (sensitivity: $52.4 \%$; specificity: $73.3 \%$ ) for GNRI to indicate mortality (Figure 1). The area under the curve was 0.644 (95\% CI: 0.516-0.772; $\mathrm{p}=0.025$ ). The highest combined sensitivity and specificity values crossed the curve at 2.5 (sensitivity: 90.5\%; specificity $89.2 \%$ ) for CONUT to indicate mortality (Figure 2). 


\section{Cureus}

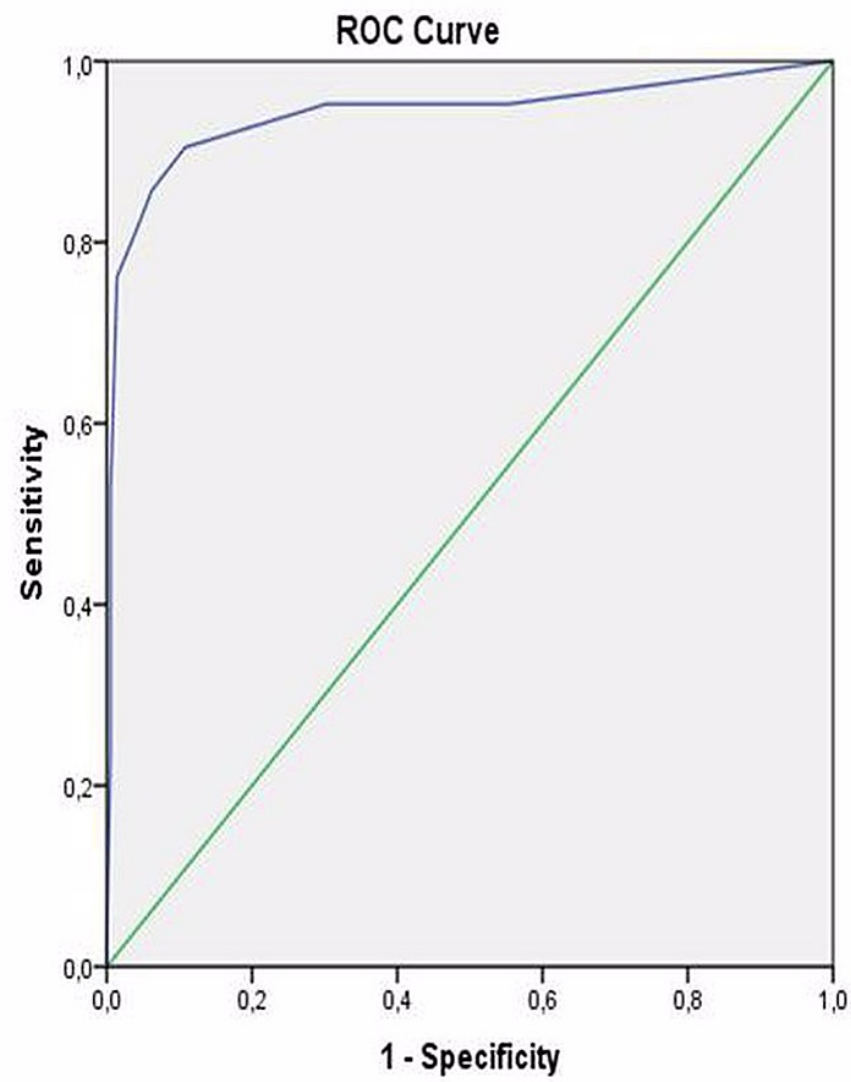

Diagonal segments are produced by ties.

FIGURE 2: ROC curve of CONUT score as a predictor of mortality

ROC: receiver operating characteristic, CONUT: controlling nutritional status

The area under the curve was 0.941 (95\% CI: 0.868-1.000; $\mathrm{p}<0.001$ ). The highest combined sensitivity and specificity values crossed the curve at 48.8 (sensitivity: $75.5 \%$; specificity: $89.1 \%$ ) for PNI to indicate MACCE (Figure 3). The area under the curve was 0.85 (95\% CI: 0.788-0.919; $<<0.001$ ). The highest combined sensitivity and specificity values crossed the curve at 59.01 (sensitivity: 69.8\%; specificity: $48.6 \%$ ) for GNRI to indicate MACCE (Figure 3). 


\section{Cureus}

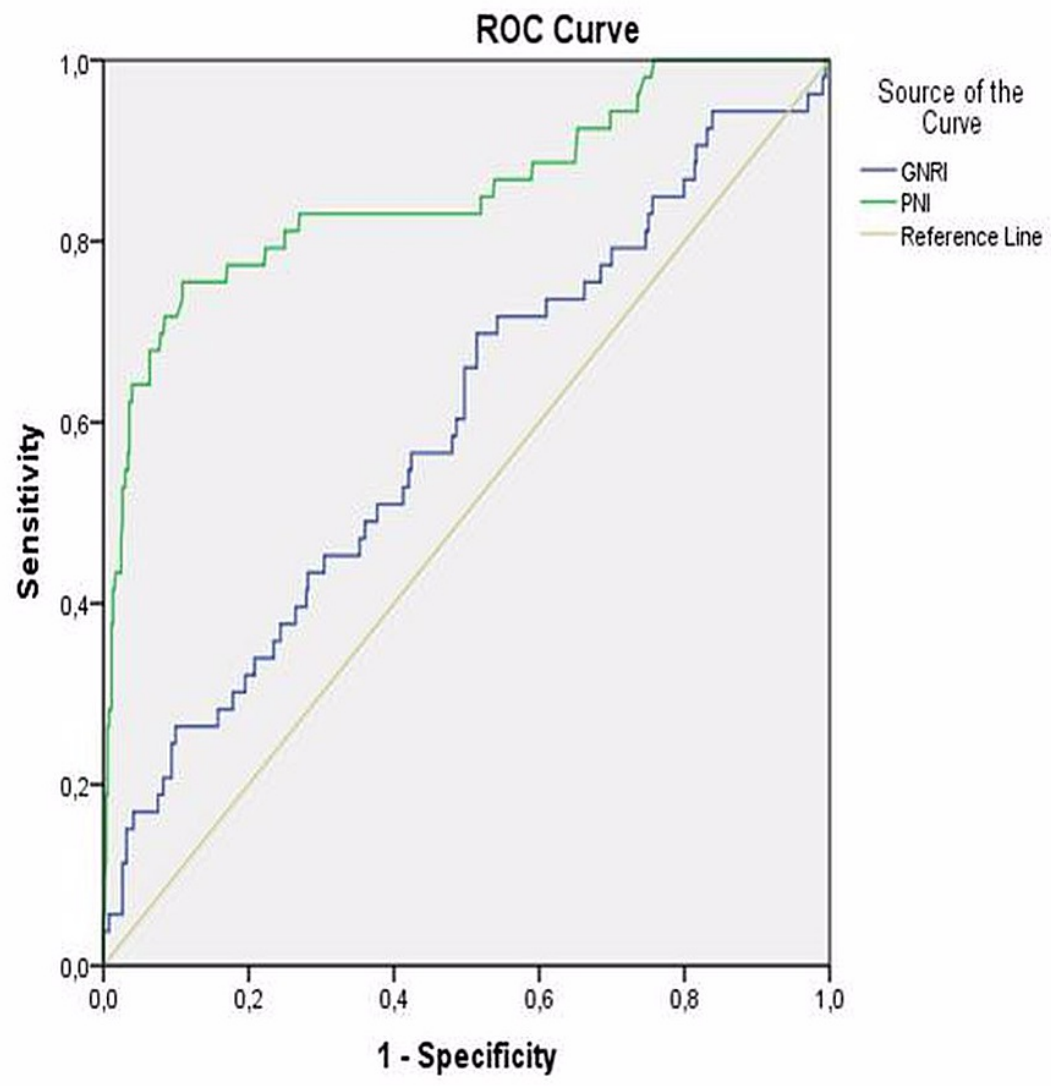

Diagonal segments are produced by ties.

FIGURE 3: ROC curves of PNI and GNRI as a predictor of MACCE

ROC: receiver operating characteristic, PNI: prognostic nutritional index, GNRI: geriatric nutritional risk index, MACCE: major adverse cardiac and cerebrovascular events

The area under the curve was 0.598 ( $95 \%$ CI: $0.514-0.681 ; \mathrm{p}=0.019$ ). The highest combined sensitivity and specificity values crossed the curve at 2.5 (sensitivity: $84.9 \%$, specificity: $93.4 \%$ ) for CONUT to indicate MACCE (Figure 4). 


\section{Cureus}

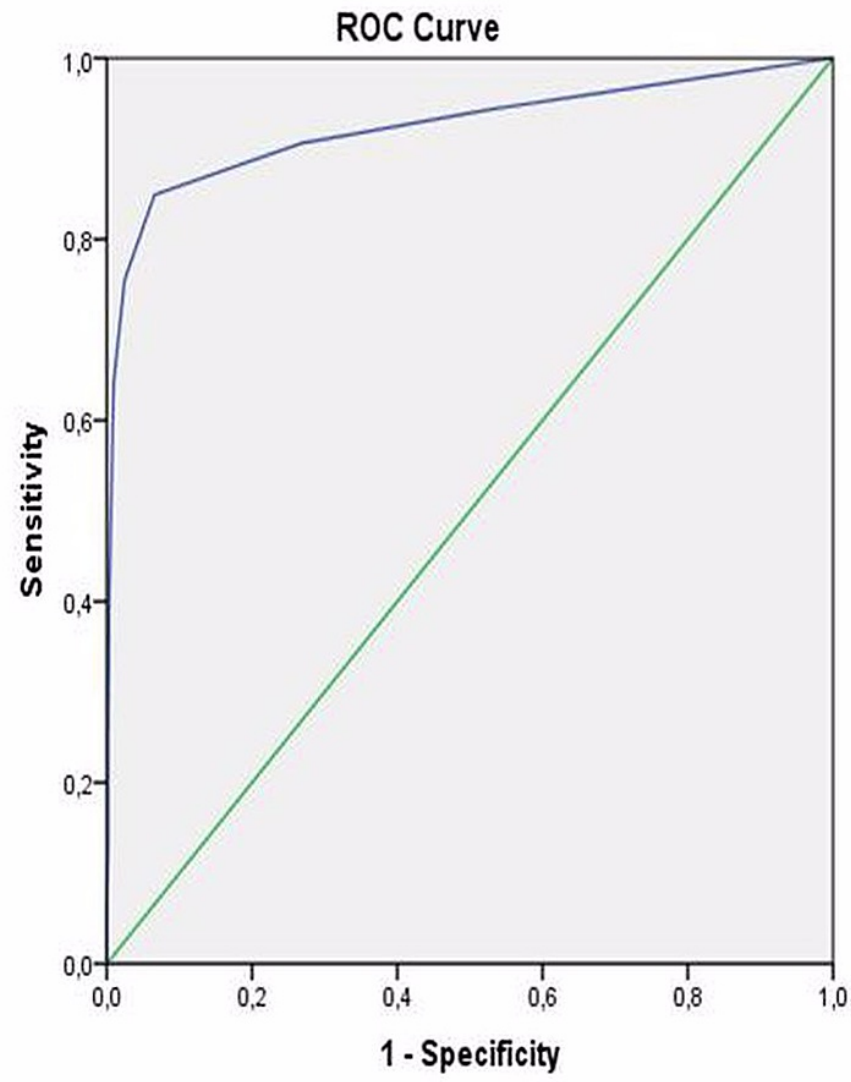

Diagonal segments are produced by ties.

\section{FIGURE 4: ROC curve of CONUT score as a predictor of MACCE}

ROC: receiver operating characteristic, CONUT: controlling nutritional status, MACCE: major adverse cardiac and cerebrovascular events

The area under the curve was 0.924 (95\% CI: 0.872 - 0.976; $\mathrm{p}<0.001)$. Then, the whole study group was divided into two groups according to their PNI and GNRI cut-off values separately. Kaplan-Meier survival analysis also revealed that long-term survival was found to be significantly decreased in patients with a lower PNI (log-rank: p<0.001) (Figure 5) and a lower GNRI (log-rank: p=0.032) (Figure 6). 


\section{Cureus}

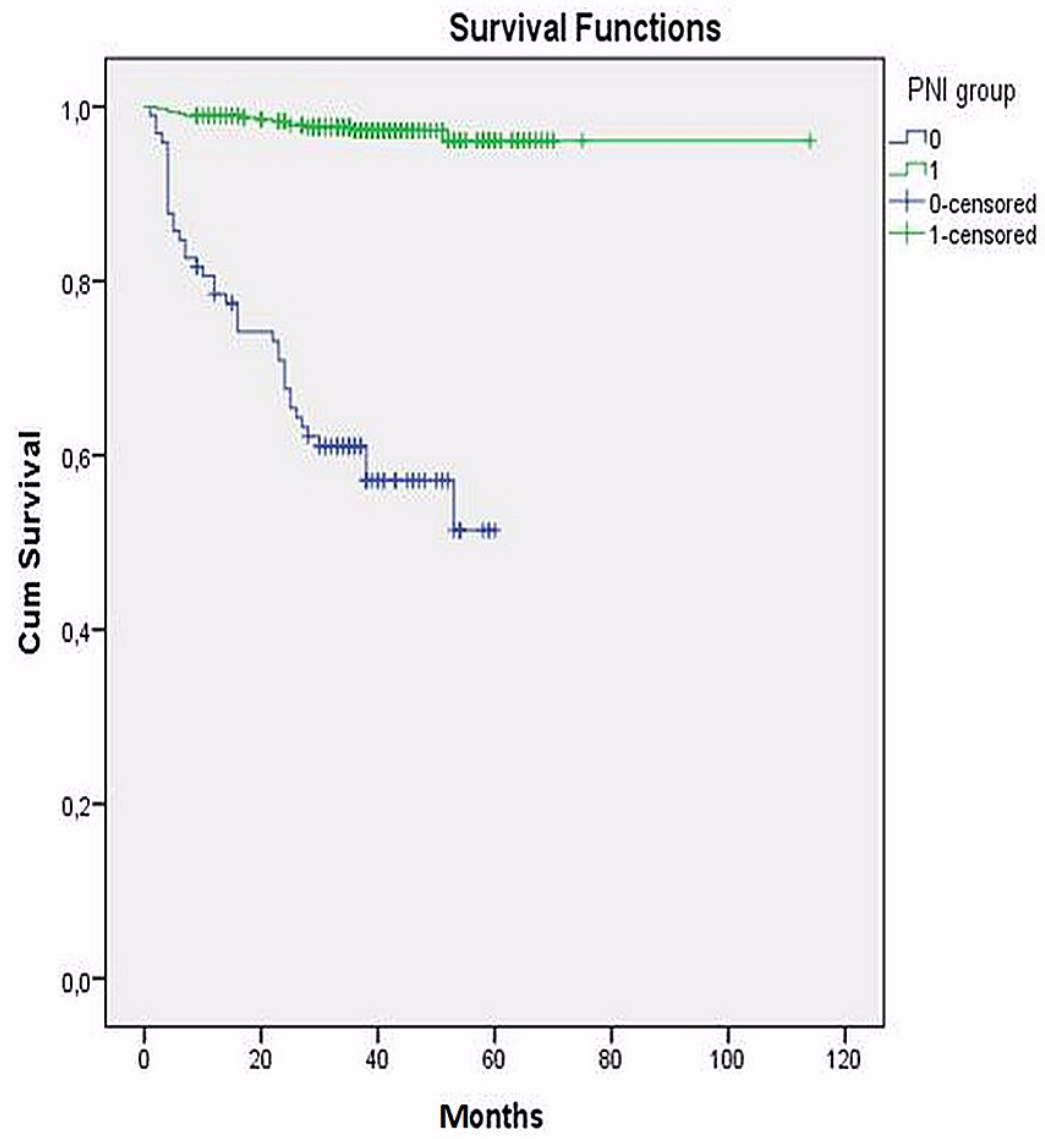

FIGURE 5: Kaplan-Meier analyses of PNI

PNI: prognostic nutritional index 


\section{Cureus}

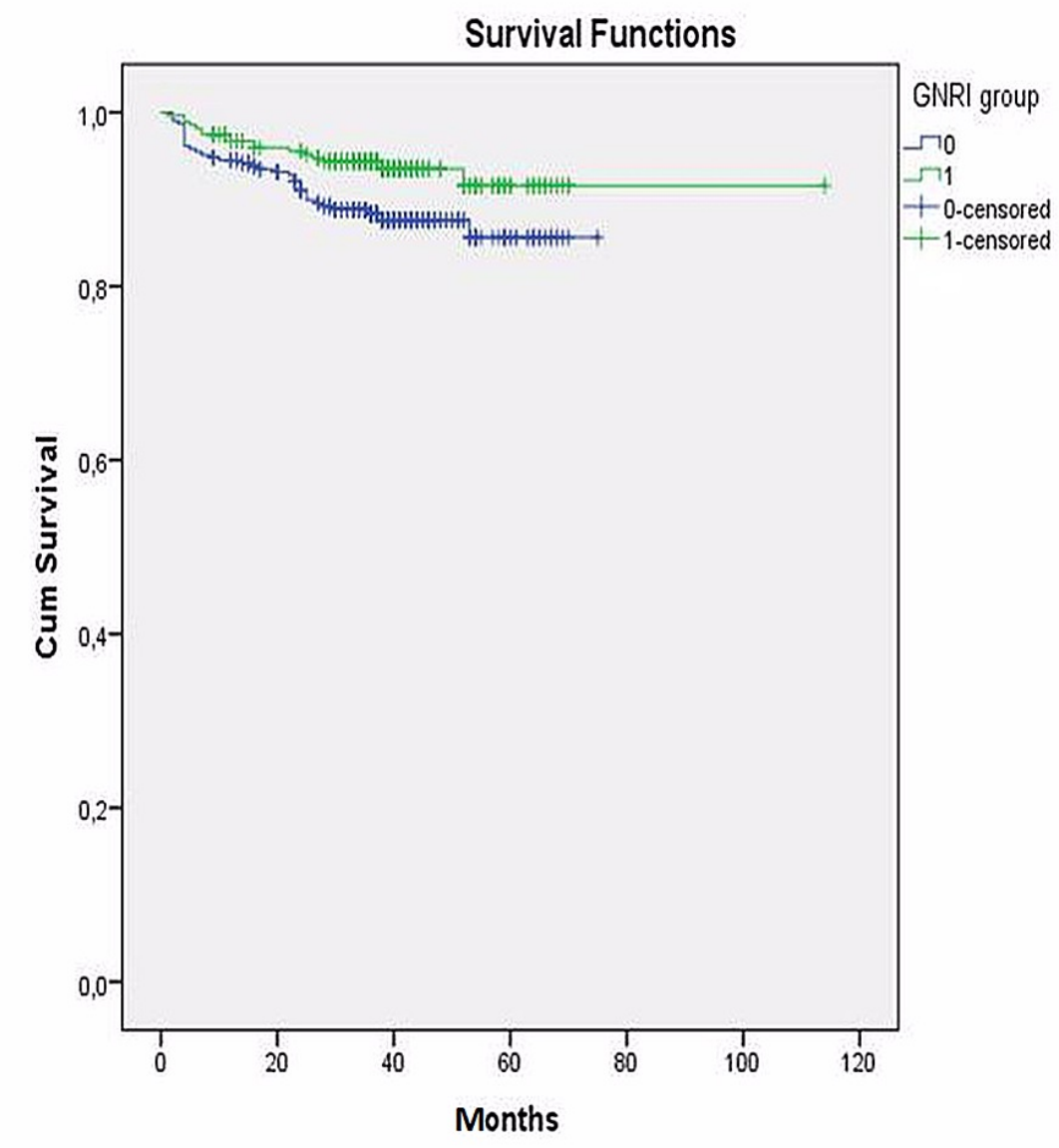

\section{FIGURE 6: Kaplan-Meier analyses of GNRI}

GNRI: geriatric nutritional risk index

Additionally, the entire study group was divided into two groups according to the CONUT value, as group 1 with normal CONUT score and group 2 with higher CONUT score indicating different level risk of malnutrition. Kaplan-Meier survival analysis also revealed that long-term survival was found significantly decreased in patients with a higher CONUT score (log-rank: p<0.001) (Figure 7). 


\section{Cureus}

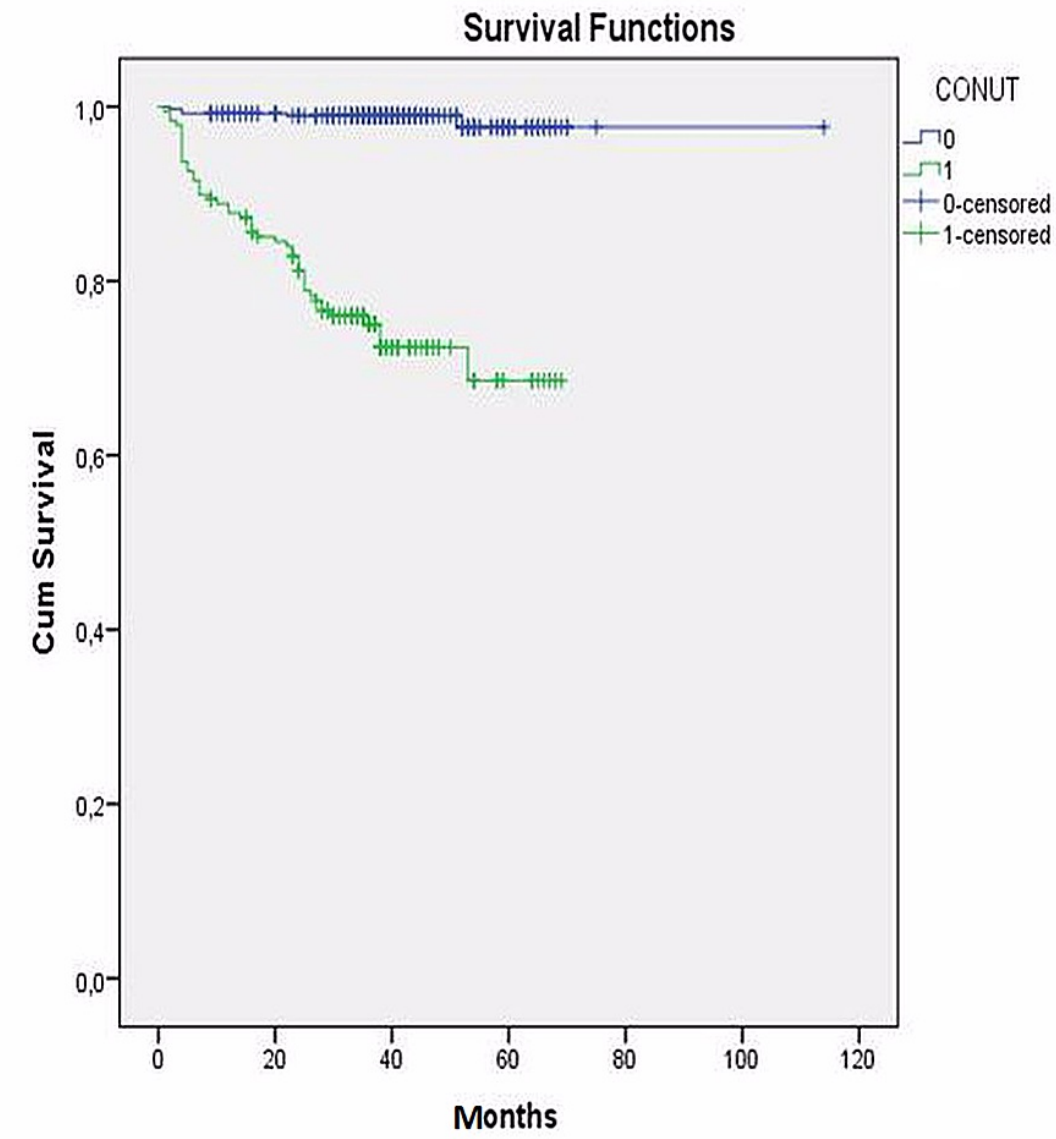

FIGURE 7: Kaplan-Meier analyses of CONUT score

CONUT: controlling nutritional status

Finally, independent predictors of MACCE were analyzed with logistic regression analyses. After logistic regression analyses, significant variables found in the univariate analysis were entered into multiple logistic regression. Two separate models were conducted for PNI and GNRI separately. The multivariate logistic regression analysis indicated that smoking (OR: $2.087 ; 95 \%$ CI: $1.040-4.188$; $\mathrm{p}=0.038$ ), PNI (OR: $0.774 ; 95 \%$ CI: $0.730-0.819 ; \mathrm{p}<0.001$ ), and PAD (OR: 3.253 ; 95\% CI: $1.250-8.462 ; \mathrm{p}=0.016$ ) were found to be independent predictors of MACCE (Table 4).

\begin{tabular}{|c|c|c|c|}
\hline & Odds ratio & $95 \%$ confidence interval & p-Value \\
\hline Smoking status & 2.087 & $1.040-4.188$ & 0.038 \\
\hline Peripheral arterial disease & 3.253 & $1.250-8.462$ & 0.016 \\
\hline Prognostic nutritional index & 0.774 & 0.730-0.819 $\quad>$ & $<0.001$ \\
\hline
\end{tabular}

TABLE 4: Multivariate analyses of parameters in predicting MACCE

MACCE: major adverse cardiac and cerebrovascular events

Additionally, smoking (OR: 3.557; 95 CI: 1.417-8.927; $p=0.007$ ), CONUT score (OR: 3.587; 95\% CI: $2.707-$ 4.752; $\mathrm{p}<0.001$ ), and PAD (OR: 4.627 95\% CI: 1.424-15.032; $\mathrm{p}=0.011$ ) were found to be independent predictors of MACCE (Table 5). 


\section{Cureus}

\begin{tabular}{|c|c|c|c|}
\hline & Odds ratio & $95 \%$ confidence interval & $p$-Value \\
\hline Smoking status & 3.557 & 1.417-8.927 & 0.007 \\
\hline Peripheral arterial disease & 4.627 & $1.424-15.032$ & 0.011 \\
\hline CONUT score & 3.587 & $2.707-4.752$ & $<0.001$ \\
\hline
\end{tabular}

TABLE 5: Multivariate analyses of parameters in predicting MACCE

CONUT: controlling nutritional status, MACCE: major adverse cardiac and cerebrovascular events

\section{Discussion}

In this study, the relationship between the objective nutritional indices and long-term MACCE in patients undergoing isolated $C A B G$ was investigated. Based on our results, we found a strong association between nutritional status and long-term MACCE and mortality rates in patients who underwent isolated CABG. Our study demonstrated that PNI, CONUT, and GNRI scores were significant prognostic nutritional markers for patients undergoing isolated CABG. This indicates that these nutritional parameters could be used as an easy and practical indicator for identifying long-term outcomes in this patient population.

With respect to the treatment of CAD, medical treatment, PCI, and open surgery are widely accepted methods, which have been valid for years $[13,14]$. Some studies have shown that CABG was a much more effective treatment modality in some patient groups $[15,16]$. Some of the biggest difficulties related to CABG are intraoperative and postoperative complications along with the development of adverse cardiovascular events. Besides, in some patients, valve surgery or additional cardiac procedures are needed who undergo CABG. Not only procedure-related risks, but the frequency of postoperative complications in these patient populations were higher than those who underwent isolated CABG [17]. Therefore, only patients who underwent isolated CABG were evaluated in our research in order to avoid long-term complications related to additional surgical procedures.

Nutritional status has a crucial role in the continuation of vital tissue and organ functions in the body. In malnutrition, which is defined as the deterioration of nutritional status, a decrease in the food intake required for the maintenance of basic organ functions, and a deterioration in the protein cycle observed [4]. Malnutrition has been reported to be associated with poor prognosis, especially in elderly patients, patients with malignancy, and patients with chronic diseases such as ischemic stroke, heart failure, or coronary artery disease [18]. This has led to the pursuit of various proposed markers in order to evaluate nutritional status. Some of these are known as PNI, CONUT, and GNRI scores. The relationship between those nutritional parameters and the development of cardiovascular diseases has been demonstrated by various studies so far $[7,9,19,20]$.

PNI, which is one of the important objective nutritional screening tools, has been used as a predictive marker for nutritional status and surgical risk in various patient populations including patients who underwent gastrointestinal surgery [11]. PNI is a marker based on the assessment of serum albumin levels and the lymphocyte count. It was shown that lower PNI levels were associated with postoperative mortality in patients undergoing CABG [11]. According to a study conducted by de Ulíbarri et al., lower PNI values were associated with higher ACE and mortality rates in patients with prior history of heart failure [12]. Outcomes of our study were compatible with the results of previous studies, which revealed lower PNI scores were associated with higher rates of MACCE in patients who underwent isolated CABG.

Another objective nutritional assessment tool is the CONUT score. This score is derived from the values of serum albumin, total cholesterol, and lymphocyte counts [12]. Albumin is an inflammation marker that plays an important role in endothelial function and lipoprotein structure. Low albumin levels may cause atherosclerotic disease through endothelial dysfunction [18]. This situation may worsen pre-existing CAD and increase the risk of future adverse cardiovascular events. The relationship between high cholesterol levels, especially elevated LDL cholesterol levels, and atherosclerotic heart disease has been known for years [19]. However, it has been reported that low LDL values in some patients also increase vascular inflammation and in some cases, this may even be an indicator of advanced atherosclerosis [20]. Malnutrition can also provoke this condition by creating low level of cholesterol concentrations [13]. Another parameter of this score is the lymphocytic count. Low lymphocyte count is an indicator of neurohormonal dysfunction in the body and is associated with low immunity in patients with CAD. This may contribute to the development of atherosclerosis by increasing vascular stress [14]. It may also be associated with poor prognosis in patients with CAD. The CONUT score was first defined by Bouillanne et al. in 2005 and found to be an important marker in terms of showing the nutritional status of hospitalized patients [10]. Studies conducted in the following years have revealed that the CONUT score can also be a useful parameter indicative of the clinical condition for patients with cardiovascular disease. CONUT score determined both 
as a factor increasing in-hospital mortality in patients with acute heart failure and chronic failure, and as a predictor of long-term mortality [12,15]. In a study by Keskin et al., CONUT score was independently associated with an increased future risk of acute myocardial infarction, cardiovascular death, heart failure, major advance cardiovascular events, and total cardiovascular events, extending the predictive value of nutrition scoring in CAD patients after PCI [5]. According to our study, MACCE and mortality were observed less frequently in patients with low CONUT scores. In addition, high CONUT score was found to be associated with worse prognosis in long-term follow-up, and lower CONUT score was associated with higher survival in patients who underwent isolated CABG.

GNRI is a nutritional evaluation method determined by objective criteria that predicts mortality and morbidity in elderly patients and patients with chronic diseases. Bouillanne et al. first described this parameter in 2005. The parameter was used as a predictive marker for the effects of complications secondary to malnutrition on in-hospital mortality at that time [10]. Apart from elderly patients, it has been shown to be an effective marker for showing nutritional status in patients less than 65 years of age. There were also studies showing that GNRI was associated with mortality in patients with prior history of heart failure [19]. According to our data, MACCE was observed more frequently in patients with isolated CABG and low GNRI scores, and lower survival was found in the long-term follow-up of these patients.

In our study, three different nutritional assessment tools were used. Gürbak et al. previously researched the outcome of all three tools on patients who underwent aortic valve replacement. Their study demonstrated the association between PNI, GNRI, CONUT score, and outcome after aortic valve replacement [21]. Similar to their study, we have also demonstrated the association of nutritional status with three different tools and cardiovascular events after isolated CABG surgery.

\section{Limitations}

There are some limitations to our study. First and foremost, the retrospective nature of the study inadvertently limited the diagnostic value of the research. Second, some factors such as intraoperative parameters that may affect the prognosis in patients undergoing CABG were not evaluated in this study. Third, some hormonal parameters may affect nutritional status, and in our study, especially serum catecholamine and steroid hormone levels were not evaluated. Due to the fact that it is a retrospective study, especially the optimal medical treatment could not be followed, this may have affected the results as a factor affecting the long-term outcomes. Furthermore, the nutritional status and malnutrition scores of the patients may change in the long-term follow-up. However, in our study, the scores calculated during the hospitalization and operation periods were considered. Because of these limitations, prospectively designed and larger studies are mandatory in order to verify these results.

\section{Conclusions}

Our study showed that nutritional indices including PNI, CONUT, and GNRI are associated with long-term MACCE and mortality in patients undergoing isolated CABG. The use of these scores in order to predict prognosis in patients treated with CABG seems to be an applicable method in clinical practice. It is important to evaluate nutritional parameters in the preoperative period in patients who will undergo isolated CABG. In this period, optimizing the nutritional status and performing surgery with the appropriate nutritional status of the patients may help to reduce postoperative and long-term outcomes. However, depending on the limitations of the study, multi-center and prospective studies are needed to validate our findings.

\section{Additional Information \\ Disclosures}

Human subjects: Consent was obtained or waived by all participants in this study. Mehmet Akif Ersoy Thoracic and Cardiovascular Surgery Training and Research Hospital Local Ethics Committee issued approval \#2020/83. Approval of the research study was accepted as exempt because the collection or study of existing data, documents, records, pathological specimens, or diagnostic specimens were recorded by the investigator in such a manner that subjects cannot be identified, directly or through identifiers linked to the subjects. Animal subjects: All authors have confirmed that this study did not involve animal subjects or tissue. Conflicts of interest: In compliance with the ICMJE uniform disclosure form, all authors declare the following: Payment/services info: All authors have declared that no financial support was received from any organization for the submitted work. Financial relationships: All authors have declared that they have no financial relationships at present or within the previous three years with any organizations that might have an interest in the submitted work. Other relationships: All authors have declared that there are no other relationships or activities that could appear to have influenced the submitted work.

\section{References}

1. Moran AE, Forouzanfar MH, Roth GA, Mensah GA, Ezzati M, Murray CJ, Naghavi M: Temporal trends in ischemic heart disease mortality in 21 world regions, 1980 to 2010: the Global Burden of Disease 2010 study. Circulation. 2014, 129:1483-92. 10.1161/CIRCULATIONAHA.113.004042 
2. Singh AK: Percutaneous coronary intervention vs coronary artery bypass grafting in the management of chronic stable angina: a critical appraisal. J Cardiovasc Dis Res. 2010, 1:54-8.

3. Brown JK, Singh K, Dumitru R, Chan E, Kim MP: The benefits of enhanced recovery after surgery programs and their application in cardiothoracic surgery. Methodist Debakey Cardiovasc J. 2018, 14:77-88.

4. Cederholm T, Jägrén C, Hellström K: Nutritional status and performance capacity in internal medical patients. Clin Nutr. 1993, 12:8-14. 10.1016/0261-5614(93)90138-t

5. Keskin M, İpek G, Aldağ M, et al.: Effect of nutritional status on mortality in patients undergoing coronary artery bypass grafting. Nutrition. 2018, 48:82-6. 10.1016/j.nut.2017.10.024

6. Açıkel ME, Korkut AK: Impact of controlling nutritional status score (CONUT) and prognostic nutritional index (PIN) on patients undergoing coronary artery bypass graft surgery. Heart Surg Forum. 2019, 22:294-7. 10.1532/hsf.2493

7. Chen SC, Yang YL, Wu CH, et al.: Association between preoperative nutritional status and clinical outcomes of patients with coronary artery disease undergoing percutaneous coronary intervention. Nutrients. 2020, 12:1295. 10.3390/nu12051295

8. Wada H, Dohi T, Miyauchi K, et al.: Combined effect of nutritional status on long-term outcomes in patients with coronary artery disease undergoing percutaneous coronary intervention. Heart Vessels. 2018, 33:144552. 10.1007/s00380-018-1201-X

9. Kunimura A, Ishii H, Uetani T, et al.: Impact of geriatric nutritional risk index on cardiovascular outcomes in patients with stable coronary artery disease. J Cardiol. 2017, 69:383-8. 10.1016/j.jjcc.2016.09.004

10. Bouillanne O, Morineau G, Dupont C, et al.: Geriatric nutritional risk index: a new index for evaluating atrisk elderly medical patients. Am J Clin Nutr. 2005, 82:777-83. 10.1093/ajcn/82.4.777

11. Buzby GP, Mullen JL, Matthews DC, Hobbs CL, Rosato EF: Prognostic nutritional index in gastrointestinal surgery. Am J Surg. 1980, 139:160-7. 10.1016/0002-9610(80)90246-9

12. De Ulíbarri JI, González-Madroño A, de Villar NG, et al.: CONUT: a tool for controlling nutritional status. First validation in a hospital population. Nutr Hosp. 2005, 20:38-45.

13. Steg PG, Ducrocq G: Future of the prevention and treatment of coronary artery disease . Circ J. 2016, 80:1067-72. 10.1253/circj.CJ-16-0266

14. Dąbrowski R, Dobrowolski M: Stable coronary artery disease - medical treatment. Pol Merkur Lekarski. 2017, 43:228-31.

15. Alexander JH, Smith PK: Coronary-artery bypass grafting. N Engl J Med. 2016, 374:1954-64. 10.1056/NEJMra1406944

16. Joseph J, Velasco A, Hage FG, Reyes E: Guidelines in review: comparison of ESC and ACC/AHA guidelines for the diagnosis and management of patients with stable coronary artery disease. J Nucl Cardiol. 2018, 25:509-15. 10.1007/s12350-017-1055-0

17. Aya HD, Cecconi M, Hamilton M, Rhodes A: Goal-directed therapy in cardiac surgery: a systematic review and meta-analysis. Br J Anaesth. 2013, 110:510-7. 10.1093/bja/aet020

18. Gomes F, Emery PW, Weekes CE: Erratum to risk of malnutrition is an independent predictor of mortality, length of hospital stay, and hospitalization costs in stroke patients. [Journal of Stroke and Cerebrovascular Diseases 2016:25(4):799-806]. J Stroke Cerebrovasc Dis. 2016, 25:2091. 10.1016/j.jstrokecerebrovasdis.2016.05.001

19. Kawamiya T, Suzuki S, Ishii H, et al.: Correlations between geriatric nutritional risk index and peripheral artery disease in elderly coronary artery disease patients. Geriatr Gerontol Int. 2017, 17:1057-62. $10.1111 /$ ggi.12828

20. Kuller LH, Eichner JE, Orchard TJ, Grandits GA, McCallum L, Tracy RP: The relation between serum albumin levels and risk of coronary heart disease in the multiple risk factor intervention trial. Am J Epidemiol. 1991, 134:1266-77. 10.1093/oxfordjournals.aje.a116030

21. Gürbak İ, Güner A, Güler A, et al.: Prognostic influence of objective nutritional indexes on mortality after surgical aortic valve replacement in elderly patients with severe aortic stenosis (from the nutrition-SAVR trial). J Card Surg. 2021, 36:1872-81. 10.1111/jocs.15434 